\title{
Recent advances in graphene family materials toxicity investigations
}

\author{
Agnieszka Maria Jastrzębska • \\ Patrycja Kurtycz $\cdot$ Andrzej Roman Olszyna
}

Received: 19 March 2012/ Accepted: 15 November 2012/Published online: 29 November 2012

(C) The Author(s) 2012. This article is published with open access at Springerlink.com

\begin{abstract}
Recently, graphene family materials (GFMs) have been introduced among all fields of science and still get numerous attention. Also, the applicability of these materials in many areas makes them very attractive. GFMs have attracted both academic and industrial interest as they can produce a dramatic improvement in materials properties at very low filler content. This article presents recent findings on GFMs toxicity properties based on the most current literature. This article studies the effects of GFMs on bacteria, mammalian cells, animals, and plants. This article also reviews in vitro and in vivo test results as well as potential anticancer activity and toxicity mechanisms of GFMs. The effect of functionalization of graphene on pacifying its strong interactions with cells and associated toxic effects was also analyzed. The authors of the article believe that further work should focus on in vitro and in vivo studies on possible interactions between GFMs and different living systems. Further research should also focus on decreasing GFMs toxicity, which still poses a great challenge
\end{abstract}

A. M. Jastrzębska ( $($ ) · P. Kurtycz · A. R. Olszyna Faculty of Materials Science and Engineering, Warsaw University of Technology, Woloska 141, 02-507 Warsaw, Poland

e-mail: agsolgala@gmail.com

P. Kurtycz

e-mail: patrycja.kurtycz@gmail.com

A. R. Olszyna

e-mail: aolszyna@meil.pw.edu.pl for in vivo biomedical applications. Consequently, the potential impact of graphene and its derivatives on humans and environmental health is a matter of academic interest. However, potential hazards sufficient for risk assessment first need to be investigated.

Keywords Graphene family materials · Graphene · Graphene oxide - Reduced graphene · Graphite ·

Toxicity $\cdot$ In vitro $\cdot$ In vivo $\cdot$ Mechanisms .

Functionalization $\cdot$ Bacteria $\cdot$ Mammalian cells . Animals

\section{Introduction}

Graphene is a newly emerging member of carbon materials with sp2-hybridized single-atom-layer structure. It is a typical two-dimensional material made of carbon atoms packed densely in a honeycomb crystal lattice. Graphene is believed to be composed of benzene rings stripped of their hydrogen atoms (Neto and Peres 2006). In 2004, Geim and coworkers successfully identified single layers of graphene and other two-dimensional crystals (Novoselov et al. 2004). Related materials include few-layer graphene, graphene nanosheets, graphene oxide, and reduced graphene oxide and can be included in graphene family materials (GFM) (Sanchez et al. 2011) (Fig. 1).

Graphene family materials have drawn much scientific attention and technological interest since their discovery due to their unique electronic and 
Fig. 1 The members of the graphene family materials: few-layered graphene (a), graphene nanosheet (b), graphene oxide $(\mathbf{c})$, and reduced graphene $(\mathbf{d})$ a
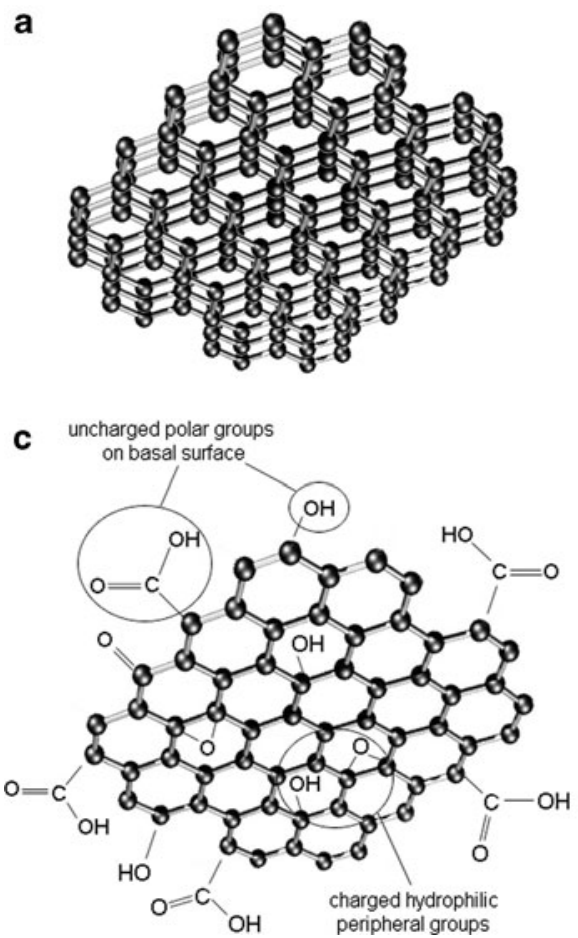

b

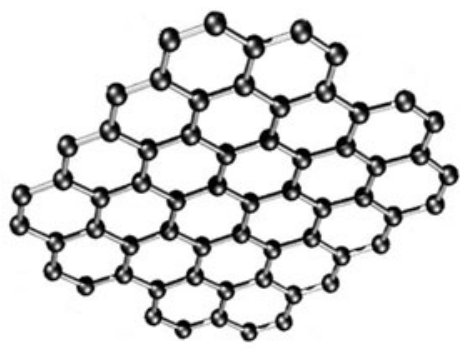

d

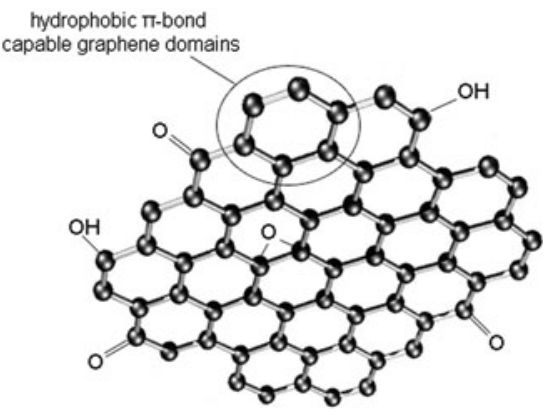

mechanical properties, specific magnetism, excellent mobility of charge carriers, and high thermal conductivity (Neto et al. 2009). High surface area, excellent conductivity, outstanding mechanical strength, and extraordinary electrocatalytic activities of these materials have also been reported in the literature (Zhang et al. 2011).

Graphene and the related materials demonstrate great potential for applications in many areas, such as field effect transistors, solar cells, sensors, and adsorbent for heavy metal removal (Li et al. 2011), lithium ion batteries, solar cells, and electrochemical super capacitors (Zhang et al. 2011).

Graphene and graphene oxide layers have also been examined in relation to building new composite materials (Wang et al. 2011). These novel nanocomposite materials have great potential for application, such as constructing electrochemical devices, energy storage devices as well as catalysts (Wang et al. 2011).

Recent studies have shown that graphene and graphene oxide exhibit several unique modes of interaction with biomolecules including preferential adsorption of single-stranded over double-stranded DNA, inter leaflet insertion in the hydrophobic core of lipid bilayers, DNA intercalation in the presence of copper cations, and high cargo carrying capacity for conjugated small molecule drugs, which can be physically adsorbed and reversibly desorbed (Sanchez et al. 2011). As a result, a number of biomedical applications have also been proposed for GFMs, with the largest set of studies focusing on graphene oxide in adsorption of enzyme, cell imaging, and drug delivery, as well as biosensors (Wang et al. 2011).

The purpose of this review is to compile up-to-date information pertaining to the biological and toxicological activity for GFMs. The aim of the review is to identify, summarize, and present information on the influence of GFM on bacteria, mammalian cells, animals, and plants on the basis of the most recent literature in the field. This article also presents the results of in vitro and in vivo tests and potential mechanisms of toxicity. Moreover, this article studies the effect of functionalization of GFMs on pacifying their strong interactions with cells and related toxic effects. A formal literature search. The article discusses the results of a formal literature review which was conducted using several international databases of scientific papers such as Science Direct, Web of Science, PubMed-NCBI, and Scirus. It has to be noted that in the case of manuscripts as well as tables and 
figures the use of the original GFMs names has been pertained. Such an approach will facilitate a detailed comparison of GFM properties.

Another aim of this review is to provide appropriate information to the scientific community so that it can be used to conduct an exposure assessment and evaluate the environmental and human health toxicity of GFMs as they are manufactured and will be introduced into the domestic market and, subsequently, the environment.

\section{Toxicity}

Toxicity to bacteria

Some studies on bacterial toxicity of GFM suggest that these materials could be used in antimicrobial products (Akhavan 2010; Hu et al. 2010). Bacterial toxicity of these materials was investigated, so far, for both Gram-positive and Gram-negative bacteria (Table 1).

Akhayan et al. (2010) researched the antibacterial activity of both graphene oxide and reduced graphene on Gram-negative Escherichia coli, and Gram-positive Staphylococcus aureus strains. The results showed that the graphene oxide reduced by hydrazine was more toxic to the bacteria than the unreduced graphene oxide. Moreover, better antibacterial activity of the reduced graphene was assigned to better charge transfer between the bacteria and more sharpened edges of the reduced graphene, during contact interaction. On the basis of measuring the efflux of cytoplasm of the bacteria, authors found that cell membrane damage of the bacteria caused by direct contact of the bacteria with the extremely sharp edges of nanowalls was an effective mechanism in bacterial inactivation. However, E. coli bacteria with an outer membrane were more resistant to the cell membrane damage than the $S$. aureus lacking the outer membrane. Similar results were obtained by $\mathrm{Hu}$ et al. (2010). They reported the antibacterial activity of graphene oxide and reduced graphene oxide only on E. coli. Within 2 h, E. coli cell metabolic activity was reduced to approximately 70 and $13 \%$ at concentrations of 20 and $85 \mu \mathrm{g} \mathrm{ml}^{-1}$, respectively. Authors also confirmed that graphene and graphene oxide produce bacterial membrane damage upon contact and caused loosing its membrane integrity.
Liu et al. (2011) compared the antibacterial activity of four types of GFMs, namely graphite, graphite oxide, graphene oxide, and reduced graphene oxide toward E. coli. The results indicated that under similar concentration $\left(40 \mu \mathrm{g} \mathrm{ml}^{-1}\right)$, graphene oxide dispersion showed the highest antibacterial activity, sequentially followed by reduced graphene oxide, graphite, and graphite oxide. SEM images suggested that cell direct contact with graphene nanosheets disrupted the cell membrane. However, no reactive oxygen species (ROS) production was detected together with the glutathione oxidization ability. The authors also concluded that antimicrobial actions resulted from both membrane and oxidation stress. Therefore, the researchers proposed a three-step antimicrobial mechanism, including: initial cell deposition on graphenebased materials, membrane stress caused by direct contact with sharp nanosheets, and the ROS-independent oxidation stress.

In comparison, three studies reported lack of graphene 41 (Zhang et al. 2011) and graphene oxide toxicity to bacteria (Wang et al. 2011; Akhavan 2012). Zhang et al. (2011) investigated graphene with a Brunauer-Emmett-Teller (BET) specific surface area of $264 \mathrm{~m}^{2} \mathrm{~g}^{-1}$ as an anodic catalyst of microbial fuel cells based on E. coli. Authors have found that lots of $E$. coli cells accumulated on the electrode surface and successfully adhered to one another with no inhibition of bacterial growth.

Wang et al. (2011) noted the lack of toxicity of graphene oxide to Shewanella species. Moreover, the graphene oxide could be reduced to graphene in a normal aerobic setup under ambient conditions as mediated by microbial respiration of Shewanella bacterial cells. Shewanella species represent an important family of dissimilatory metal-reducing bacteria, which can transfer metabolically generated electrons from a cell interior to external electron acceptors, such as solid metal oxides during anaerobic respiration. Extracellular electron transfer pathways at the cell/graphene oxide interface were systematically investigated by the authors, suggesting that both direct electron transfer and electron mediators are involved in the graphene oxide reduction.

In a very recent study, Akhavan et al. (2012) examined interactions of chemically exfoliated graphene oxide nanosheets and E. coli species living in mixed-acid fermentation environment and anaerobic conditions. By an XPS method, authors found that 


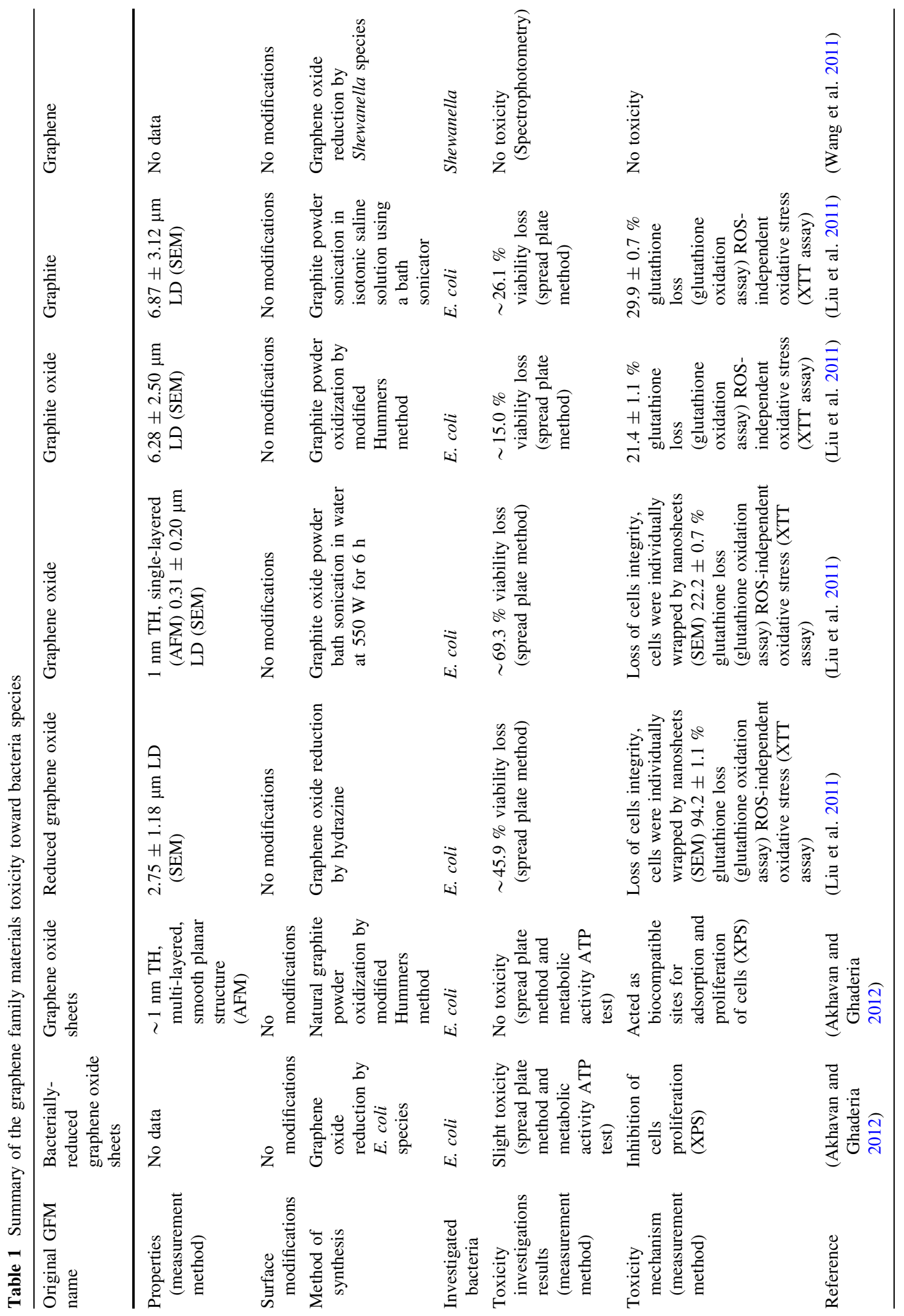




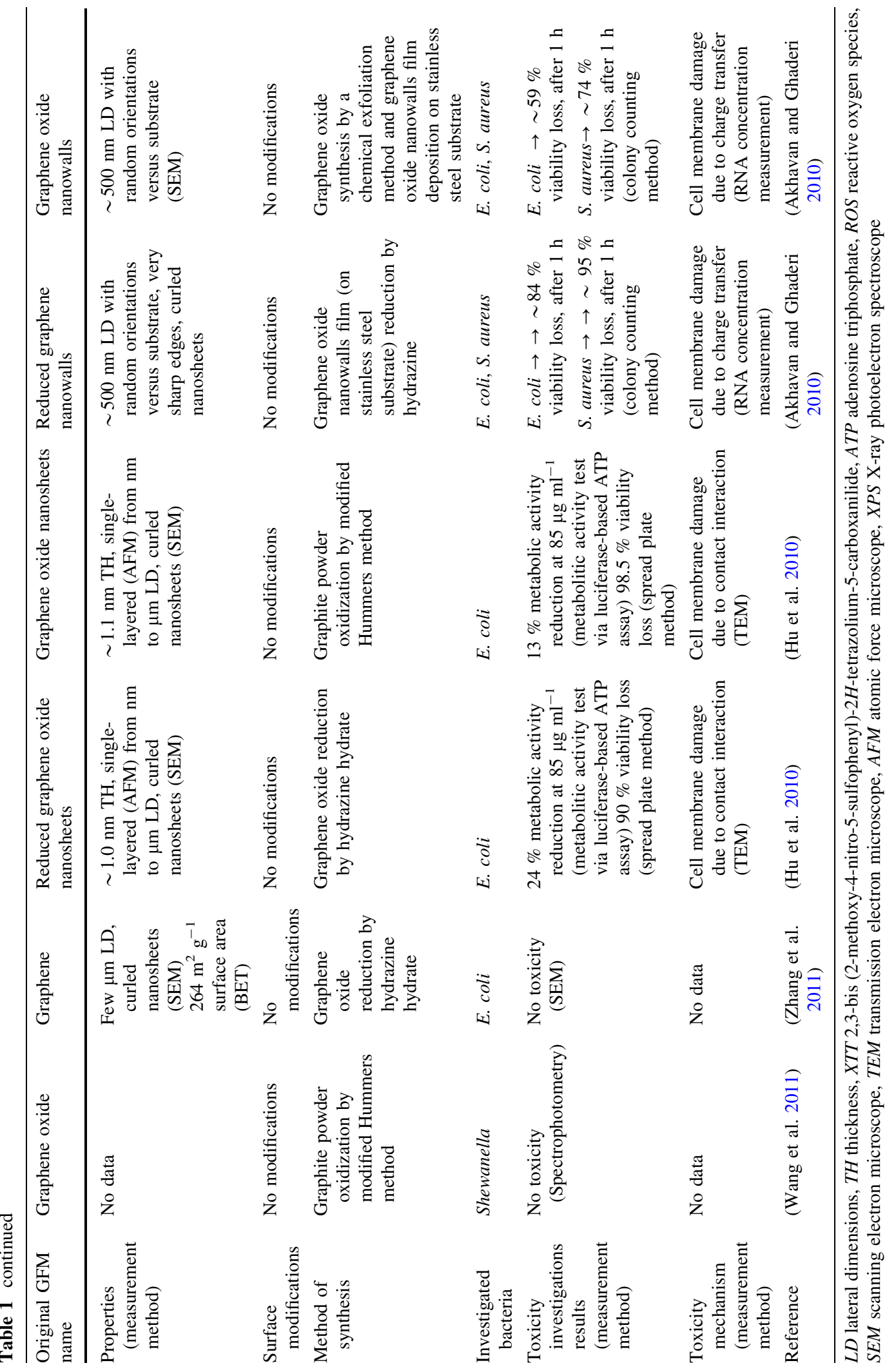


E. coli reduced graphene oxide to bacterially reduced graphene in a self-limiting manner. Graphene oxide sheets acted as biocompatible sites for adsorption and proliferation of bacteria cells on their surfaces, while the bacterially reduced graphene oxide sheets showed an inhibition for proliferation of the bacteria on their surfaces. Authors showed that the slight antibacterial property of the bacterially reduced graphene oxide sheets and the detaching of the already proliferated bacteria from their surface contributed to the growth inhibition of the bacteria on the surface of the reduced sheets.

\section{In vitro toxicity}

The interaction between dispersed GFM has been studied in vitro using human cell cultures, such as fibroblasts (Wang 2011), epithelial cells (Chang et al. 2011), alveolar basal epithelial cells (Hu et al. 2011), pheochromocytoma cells, oligodendroglia cells, fetal osteoblasts (Agarwal et al. 2010), cervical cells (Gollavelli and Ling 2012), skin fibroblasts (Liao et al. 2011), red blood cells (Liao et al. 2011), epithelial breast cancer cells (Robinson et al. 2011) as well as neuronal cells (Zhang et al. 2010). Mouse neuronal (Li et al. 2011) and pheochromocytoma cells (Agarwal et al. 2010) were also analyzed and the available in vitro toxicity literature data has been summarized in Table 2.

Literature data indicate that exposure to GFM may induce severe cytotoxicity and lung diseases. Wang et al. (2011) demonstrated that graphene oxide could produce cytotoxicity in dose- and time-dependent means, and can enter human lung fibroblasts cytoplasm and nucleus, decreasing cell adhesion, and inducing cell floating and apoptosis at doses above $20 \mu \mathrm{g} \mathrm{ml}^{-1}$ after $24 \mathrm{~h}$. The results indicated that graphene oxide of dose less than $20 \mu \mathrm{g} \mathrm{ml}^{-1}$ failed to exhibit toxicity to human fibroblast cells, while the dose of more than $50 \mu \mathrm{g} \mathrm{ml}^{-1}$ exhibited obvious cytotoxicity reflected in decreasing cell adhesion or inducing cell apoptosis during 1-5 days following cell seeding. Authors also confirmed that GFM can enter the lung tissues and stop there and induce lung inflammation and subsequent granulomas highly dependent on injected dose.

Chang et al. (2011) also investigated toxicity of graphene oxide by examining its influence on the morphology, viability, mortality, and membrane integrity of human lung epithelial cells. However, the results suggested that graphene oxide did not enter cells and had no obvious cytotoxicity. Authors found out that graphene oxide could only cause a slight dosedependent oxidative stress in cell and induce a slight loss of cell viability even at the concentration of $50 \mu \mathrm{g} \mathrm{ml}^{-1}$.

Hu et al. (2011) have also carried out a systematic study on cellular effects of graphene oxide. Authors observed that human alveolar basal epithelial cells (A549) were sensitive to the presence of graphene oxide and showed concentration-dependent cytotoxicity.

Zhang et al. (2010) proved that graphene could induce cytotoxic effects and mitochondrial injury in human neuronal cells after 4 and $24 \mathrm{~h}$ at a dose of $10 \mu \mathrm{g} \mathrm{ml}^{-1}$. The effects observed in the examination were concentration- and shape-dependent. Interestingly, at low concentrations, graphene induced stronger metabolic activity than carbon nanotubes, and this trend was, however, reversed at higher concentrations. Furthermore, time-dependent caspase 3 activation after exposure to graphene $\left(10 \mu \mathrm{g} \mathrm{ml}^{-1}\right)$ showed the evidence of neuronal cells apoptosis. Gollavelli and Ling (2012) studied in vitro cytotoxicity of graphene to human cervical cancer cells (HeLa). The results suggested that graphene exhibited toxicity with an IC50 value of $\sim 100 \mathrm{mg} \mathrm{ml}^{-1}$.

Liao et al. (2011) showed that the toxicity of graphene and graphene oxide depends on the exposure environment (i.e., whether or not GFM aggregation occurs) and mode of interaction with cells. Authors explored the toxicity of GFM toward human red blood cells and skin fibroblasts. The greatest hemolytic activity was displayed by the graphene oxide, whereas aggregated graphene sheets exhibited the lowest hemolytic properties. Water-soluble tetrazolium salt (WST-8), trypan blue exclusion and ROS assays revealed that the graphene sheets were more damaging to mammalian fibroblasts than the graphene oxide and generated significant amount of ROS in human skin fibroblast cells. These GFMs also strongly bound to the cell surface.

Schinwald et al. (2012) also demonstrated that the layered (1-10 layers) graphene nanoplatelets exceeding a size of approximately $15 \mu \mathrm{m}$ projected diameter could not be fully phagocytosed by immortalized human monocytic (THP-1) cells which led to inhibition of phagocytosis process and frustrated phagocytosis occurrence. Authors also found that concentrations of $5 \mu \mathrm{g} \mathrm{cm}^{-2}$ and higher significantly increased the LDH 


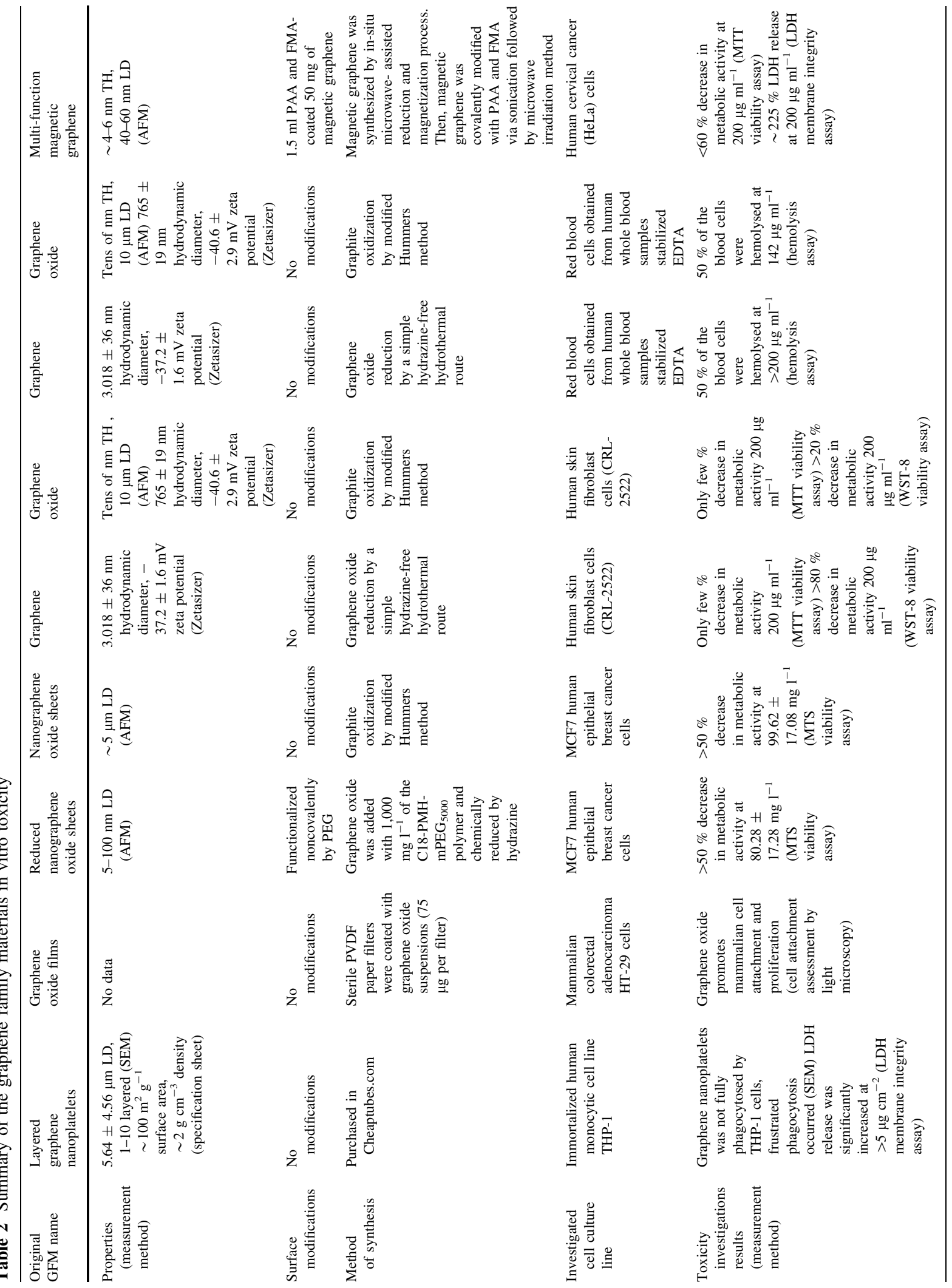




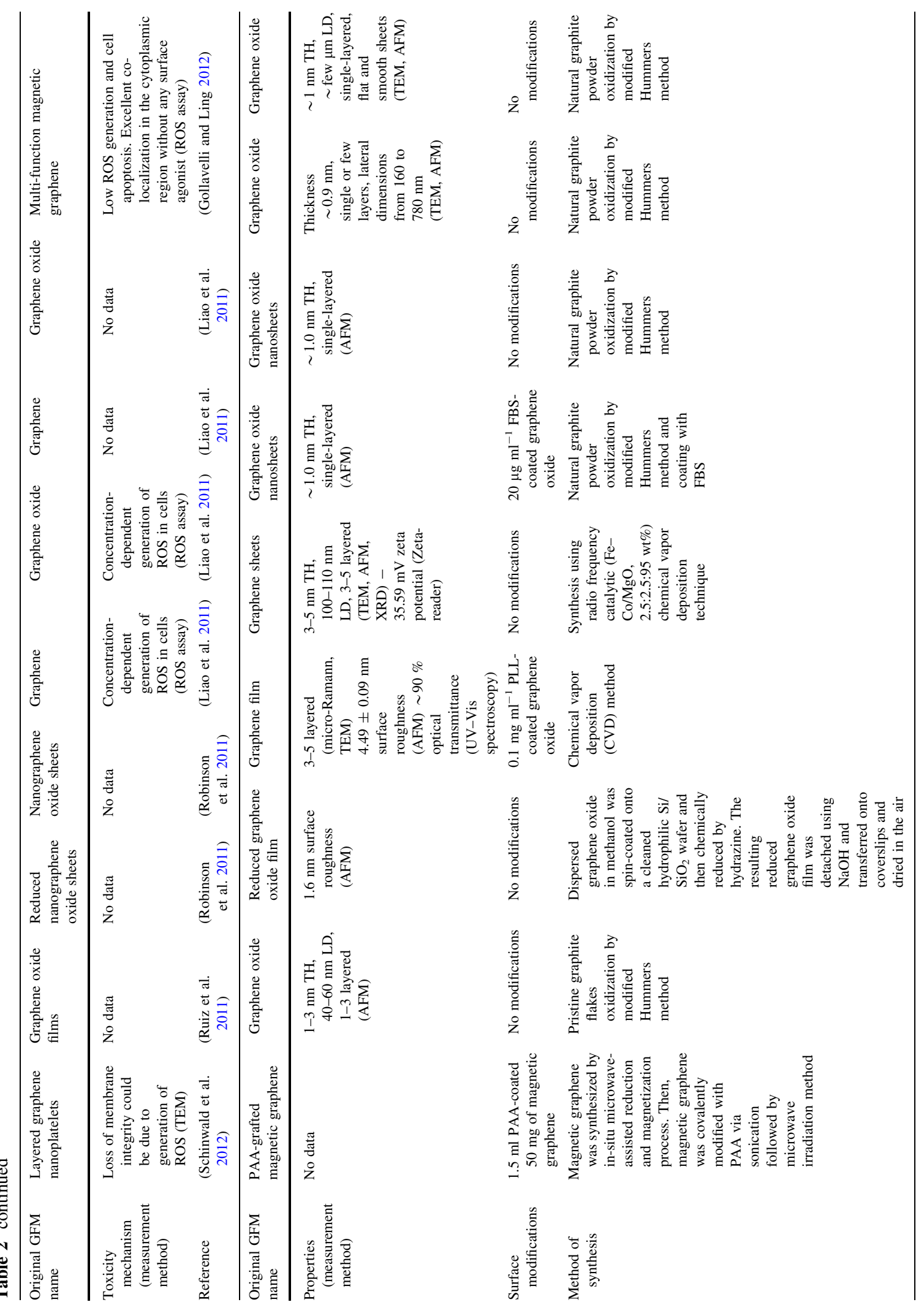




\begin{tabular}{|c|c|c|c|}
\hline 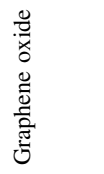 & 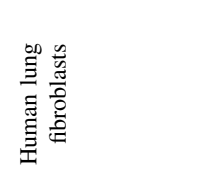 & 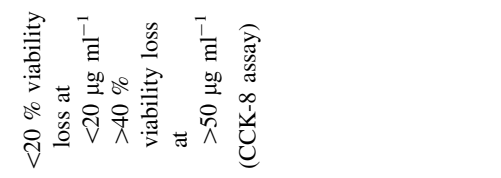 & 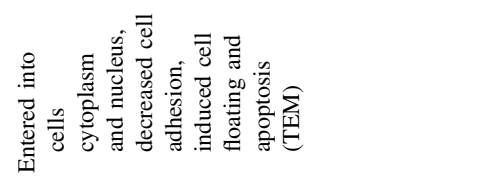 \\
\hline 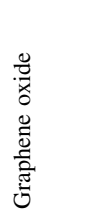 & 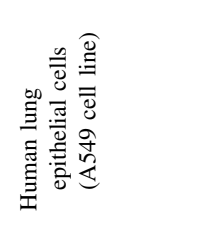 & 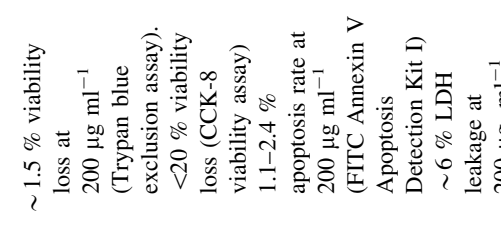 & 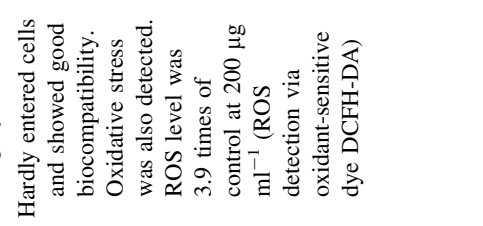 \\
\hline 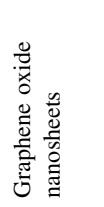 & 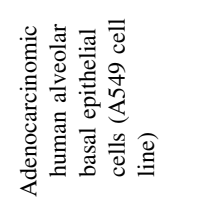 & 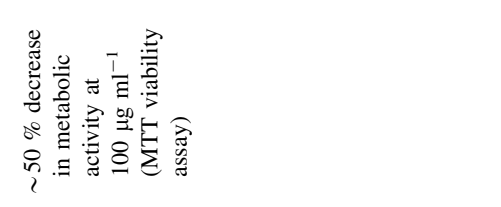 & 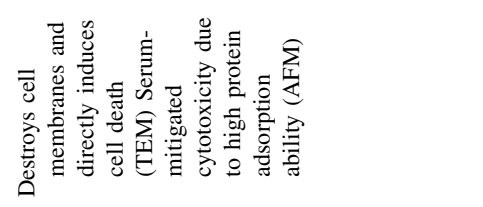 \\
\hline 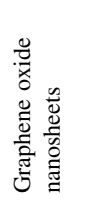 & 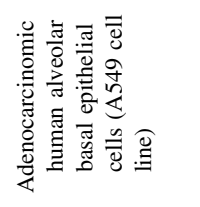 & 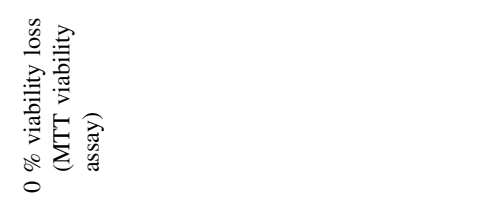 & 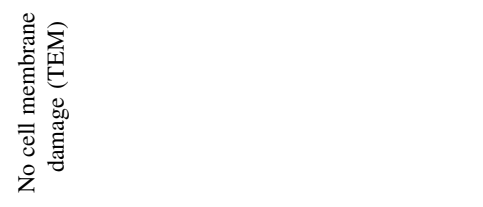 \\
\hline & 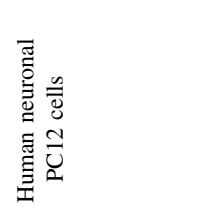 & 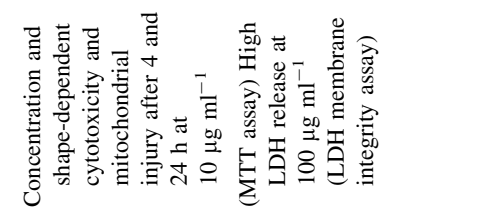 & 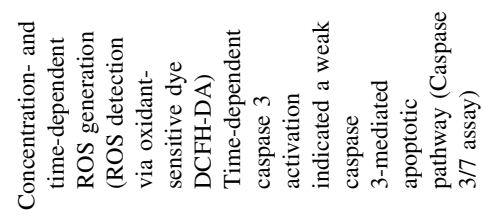 \\
\hline 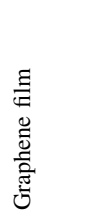 & 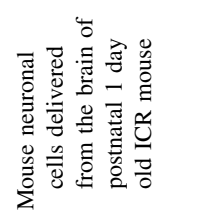 & 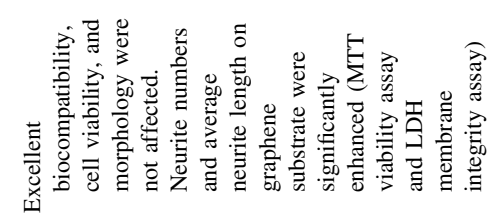 & 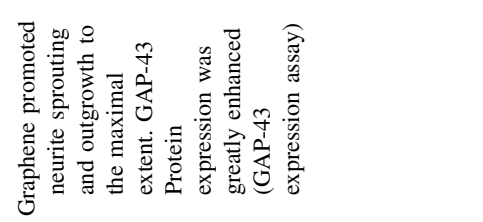 \\
\hline 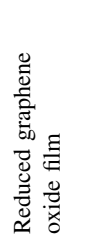 & 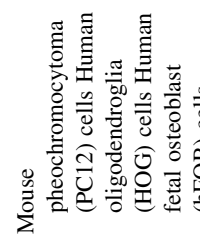 & 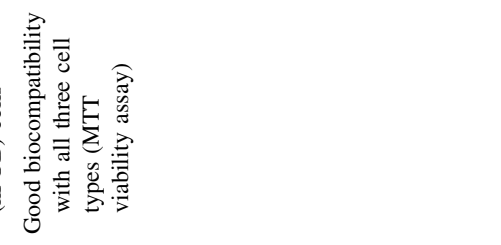 & 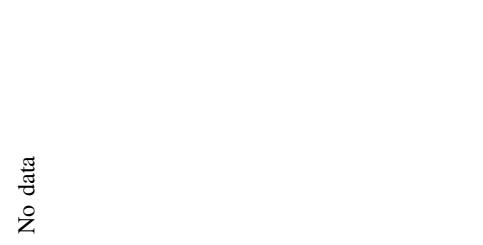 \\
\hline 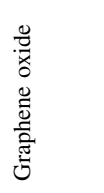 & 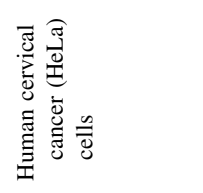 & 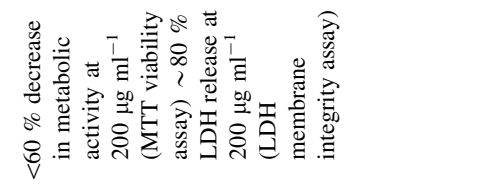 & 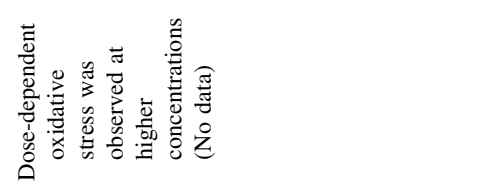 \\
\hline 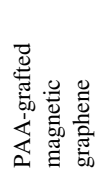 & 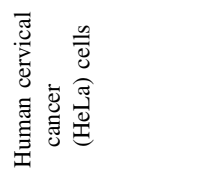 & 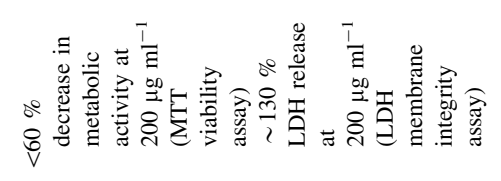 & 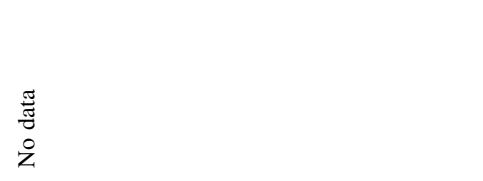 \\
\hline 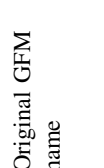 & 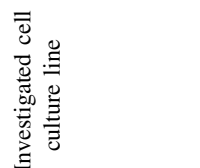 & 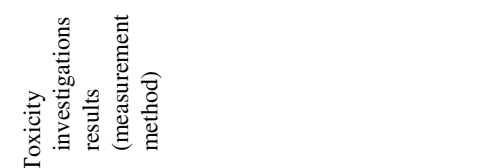 & 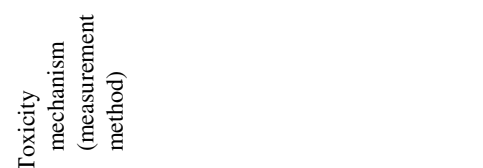 \\
\hline
\end{tabular}


release resulting in loss of membrane integrity and decrease in macrophages viability. The loss of membrane integrity could be a result of generation of ROS.

In comparison with these studies, some results have shown that GFMs in the form of film can exhibit excellent biocompatibility with no viability inhibition of investigated cells.

Reduced graphene oxide in the form of a film was found to be non-toxic to the cells examined. Agarwal et al. (2010) studied the ability of reduced graphene oxide films in inducing toxic effects in three types of cells, such as mouse pheochromocytoma cells, human oligodendroglia cells, and human fetal osteoblasts. The authors found that reduced graphene oxide showed good biocompatibility with all these cell types.

Ruiz et al. (2011) also studied the role of graphene oxide film (glass slides coated with $10 \mu \mathrm{g}$ of graphene oxide) on mammalian colorectal adenocarcinoma HT29 cells attachment and proliferation using light microscopy. The results indicated that the mammalian cells were attached more efficiently to the graphene oxide films with no damage on cells morphology or enlargement. These results clearly showed that the graphene oxide films exhibited no toxicity to the investigated cells and actually promoted their attachment and proliferation.

Similar results were obtained by $\mathrm{Li}$ et al. (2011). Authors observed good biocompatibility of graphene films toward mouse neuronal cells. Authors observed that cells numbers and average neurite length on graphene films were significantly enhanced during 2-7 days following cell seeding. These results suggested that graphene could efficiently promote neuronal cells growth. However, it should be noted that these films were additionally coated with PLL which makes these results difficult to compare with other results.

\section{In vivo toxicity}

Only five studies reported biodistribution and toxicity of graphene oxide following intravenous and intratracheal injection in mice (Table 3). Wang et al. (2011) divided thirty Kun Ming mice into three test groups (low, middle, high dose) and one control group. Test groups were injected intravenously with $0.1,0.25$, and $0.4 \mathrm{mg}$ graphene oxides, respectively. Graphene oxide under low dose $(0.1 \mathrm{mg})$ and middle dose $(0.25 \mathrm{mg})$ did not exhibit visible toxicity to mice and under high 
dose $(0.4 \mathrm{mg}$ ) exhibited chronic toxicity (4 out of 9 mice died). At a dose of $0.4 \mathrm{mg}$ graphene oxide caused granuloma formation, in the kidneys, lungs, liver, spleen, and could not be cleaned by kidney. At a dose of $0.4 \mathrm{mg}$ graphene oxide was not filtrated by the kidneys.

Similar results were obtained by Zhang et al. (2011) who investigated the distribution and biocompatibility of graphene oxide in Kun Ming mice. The use of radiotracer technique revealed high uptake and long term retention of graphene oxide in the lungs as well as a relatively long blood circulation time. No significant pathological changes in all the examined organs were observed following the exposure to $1 \mathrm{mg} \mathrm{kg}^{-1}$ of graphene oxide for 14 days. However, 10-fold increase of the dose led to forming significant pathological changes. Following the exposure to $10 \mathrm{mg} \mathrm{kg}^{-1}$ body weight of graphene oxide for 14 days, authors observed significant pathological changes, such as inflammation, cell infiltration, pulmonary edema, and granuloma formation in the lungs of mice.

Duch et al. (2011) administered the solutions of pristine graphene, Pluronic (block copolymer) dispersed graphene, and graphene oxide directly into the lungs of six C57BL mice. The introduction of graphene oxide resulted in severe and persistent lung injury. The examination of the lung tissues revealed an increased rate of mitochondrial respiration and the generation of ROS as well as the presence of activated inflammatory and apoptotic pathways.

As for in vivo studies on the GFM toxicity to other living organisms, Gollavelli and Ling experimented on fish (Gollavelli and Ling 2012) and Zanni experimented on nematodes (Zanni et al. 2012). Gollavelli and Ling (2012) studied in vivo cytotoxicity of GFM to Danio rerio (zebrafish) embryos microinjected with multi-function graphene (coated with PAA and FMA). The studies proved that this material was biocompatible with zebrafish and failed to induce any significant abnormalities or affect the survival rate of fish embryos. Confocal laser scanning microscopy images revealed that multi-function graphene was located only in the embryo' cytoplasm region and exhibited good biodistribution from the head to tail in the zebrafish. However, it should be noted that the multi-function graphene used in the study was coated with PAA and PLL which could lead to the lack of toxicity of the material.
Zanni et al. (2012) evaluated the toxicity of graphite nanoplatelets in the model living organism such as Caenorhabditis elegans (nematode). The absence of any acute or chronic toxicity of GNPs was observed. The authors examined longevity (life expectancy) as well as reproductive capability end points. Moreover, no effect on $C$. elegans reproductive potential was found. Good spatial distribution of the GFM inside the nematodes was demonstrated with the use of Fouriertransform infrared spectroscopy (FT-IR) mapping.

\section{Potential mechanisms of toxicity}

Uncertainty still prevails as to toxicity pathways for GFM. Two-dimensional graphene nanomaterials are unique in comparison with spherical nanoparticles or one-dimensional nanotubes or nanorods, and the chemical and physical determinants for their cellular interactions and biocompatibility are still under studies (Sanchez et al. 2011). Direct or indirect generation of ROS leading to oxidative stress in target cells is currently the main mechanism proposed for the toxicity of engineered nanomaterials (Oberdörster and Oberdörster 2005; Stone and Schins 2009). Some reports also indicate that generation of ROS in target cells is a potential mechanism for graphene toxicity [14]. It should be also noted that cellular homeostasis process produces a balance between the level of ROS generation and its elimination or reduction by antioxidant enzymes. The level of ROS is balanced by the action of superoxide dismutase, catalase, or glutathione peroxidase. When it cannot be reduced by cellular antioxidant activity, this may lead to alteration of macromolecules such as polyunsaturated fatty acids in membrane lipids, protein denaturation, and ultimately DNA destruction (Sanchez et al. 2011). If the level of ROS is not reduced by cellular antioxidant activity, the alteration of macromolecules such as polyunsaturated fatty acids in membrane lipids, protein denaturation, and ultimately DNA destruction may occur (Sanchez et al. 2011). Thus, the presence of extremely high hydrophobic surface areas in some GFMs may result in significant interactions with membrane lipids. This may lead in turn to direct physical toxicity or adsorption of biological molecules leading to indirect toxicity (Sanchez et al. 2011). Moreover, some studies suggest that ROS are generated in a concentration- and timedependent manner after exposure to GFM, indicating an oxidative stress mechanism (Zhang et al. 2010). 


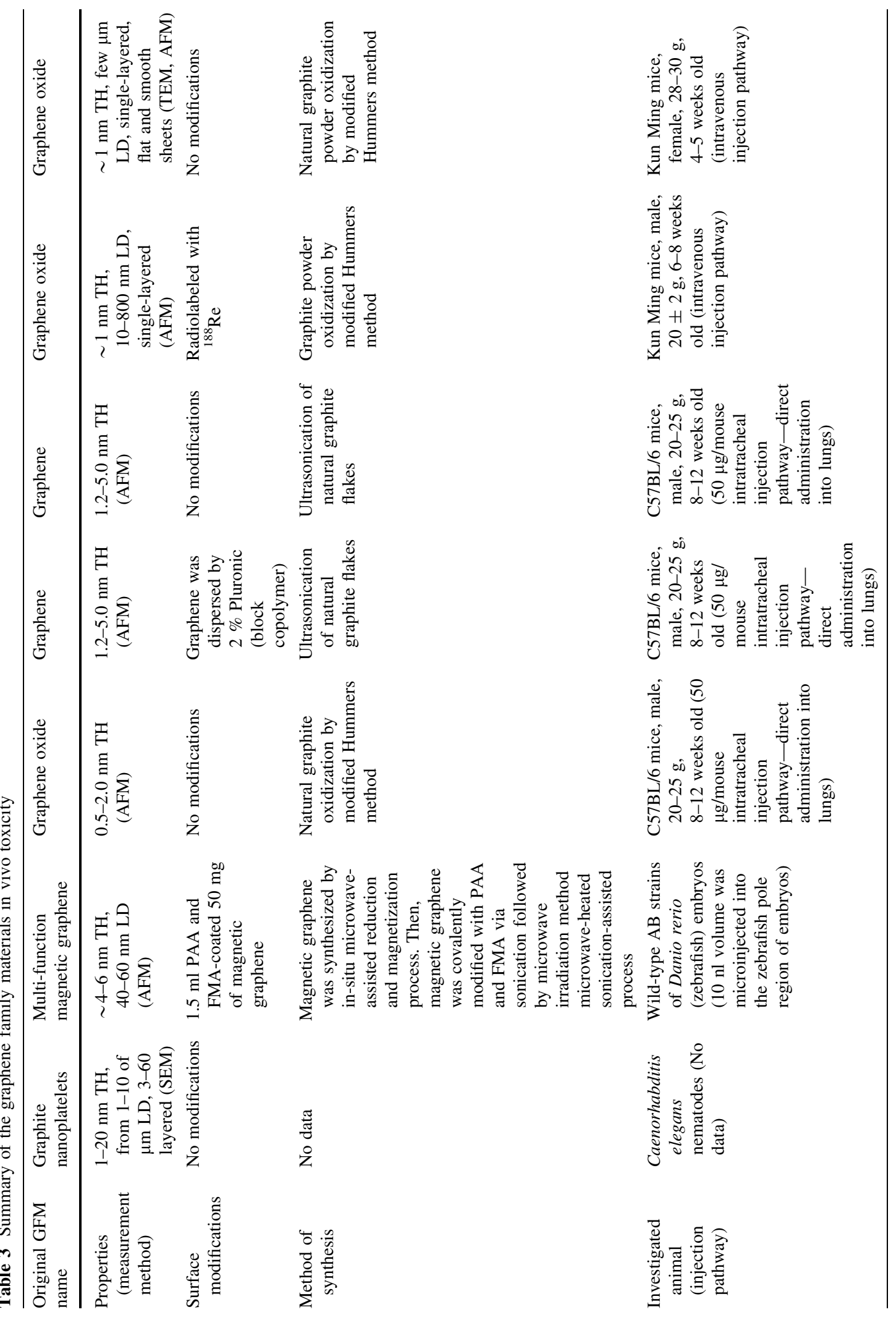




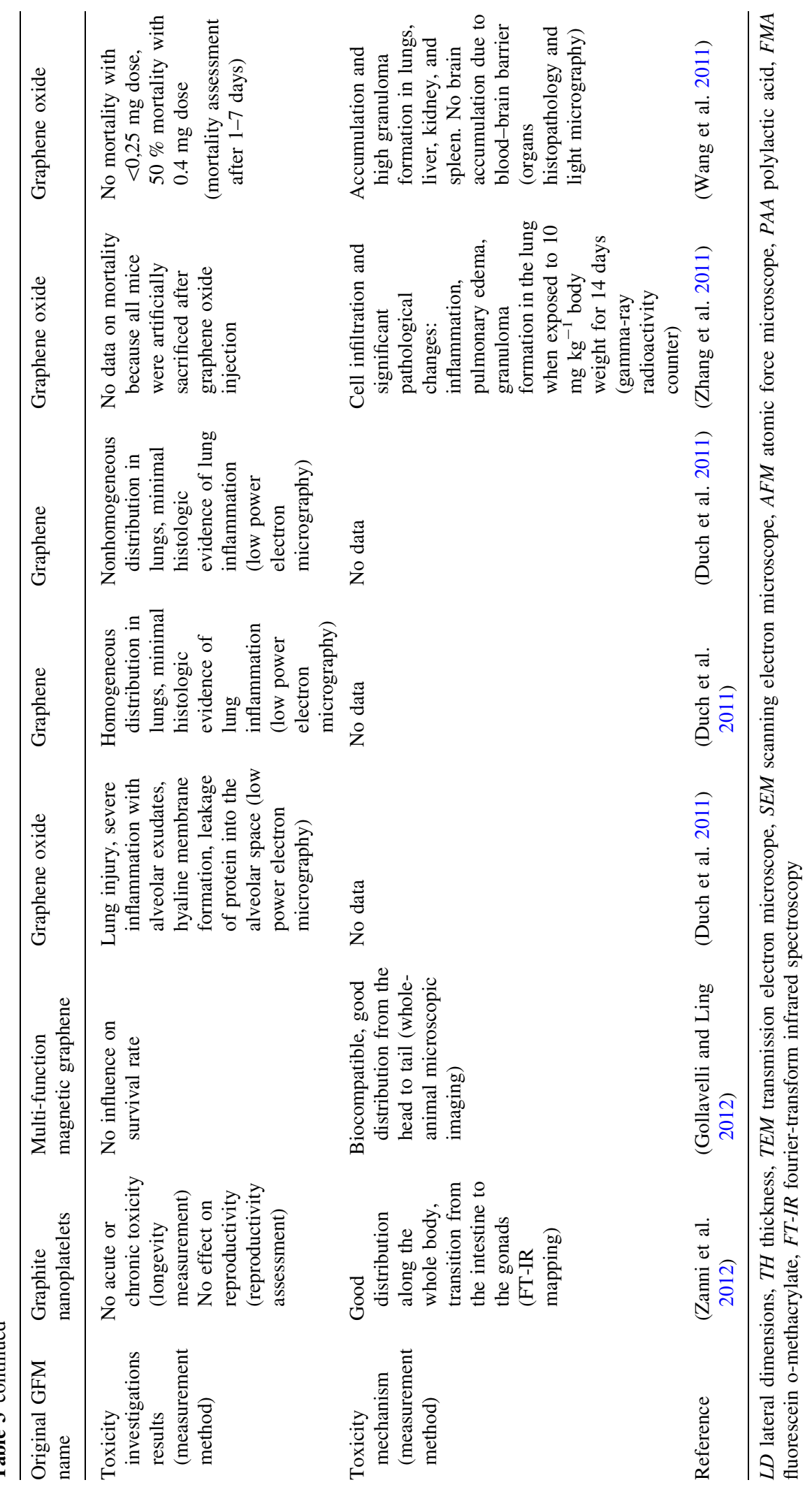


Unfortunately, the results in the literature are inconsistent, particularly concerning the ability of graphene to enter the cells. For example, Wang et al. (2011) demonstrated that graphene oxide can enter the cytoplasm and nucleus of human lung epithelial cells or fibroblasts, decreasing cell adhesion and inducing cell floating and apoptosis. In contrast, Chang et al. (2011) reported that graphene oxide cannot enter human alveolar basal epithelial cells and has no obvious cytotoxicity.

In comparison to the studies mentioned above, $\mathrm{Hu}$ et al. (2011) observed that the cytotoxicity of graphene oxide which resulted from direct interactions between the cell membrane and graphene oxide led to physical damage in the cell membrane. The damage was triggered off by interactions between the cell membrane and graphene oxide nanosheets. Interestingly, the authors discovered that the cytotoxicity of graphene oxide nanosheets occurred mostly during the initial contact stage of graphene oxide and cells and was independent of exposure duration. Physical damage of the cell membrane observation, however, excludes the contribution of an oxidative stress mechanism since that is a time-dependent process. Similar results were obtained by $\mathrm{Hu}$ et al. in the previous studies ( $\mathrm{Hu}$ et al. 2010). The research suggests that graphene oxide and reduced graphene oxide produced bacterial (E. coli) membrane damage upon direct contact. The authors confirmed these results using transmission electron microscopy, which revealed that the bacterial cells lost their membrane integrity.

By the close look at cellular functions at proteome level, Yuan et al. (2011) clearly identified the distinct pattern of cellular responses between graphene-treated cells. Overall 37 differentially expressed proteins involved in metabolic pathway, redox regulation, cytoskeleton formation, and cell growth were identified by the authors. On the basis of the protein profile, authors successfully identified the key enzymes involved in the redox processes regulation of the cell, and suggested that graphene did not trigger the upregulation of the thioredoxin-peroxiredoxin system to counter the ROS stress or did not induce the apoptosis based on the protein profile. Li et al. (2012) also demonstrated that graphene induced cytotoxicity through the depletion of the mitochondrial membrane potential and the increase of intracellular ROS. The studies also suggest that graphene can trigger apoptosis by mitochondrial pathway activation. The MAPKs (JNK, ERK and p38) as well as the TGF-betarelated signaling pathways were activated in the graphene-treated cells, which in turn activated Bim and Bax, two pro-apoptotic member of Bcl-2 protein family. Consequently, the caspase 3 and its downstream effector proteins were activated and the execution of apoptosis was initiated.

\section{Toxicity versus functionalization}

Several studies attempted to address the interactions of graphene and its derivatives with different molecules. There some evidence which proves that polymer chains, drugs, and targeting molecules can be covalently attached to the graphene surface and edge site, or polymers may be adsorbed onto the graphene surface to enhance solubility and biocompatibility (Yan et al. 2011).

Hu et al. (2011) carried out a systematic study on cellular effects of graphene oxide nanosheets and identified the effect of fetal bovine serum (FBS), an often-employed component in cell culture medium, on the cytotoxicity of graphene oxide. At low concentrations of FBS (1\%), human cells were sensitive to the presence of graphene oxide and showed concentration-dependent cytotoxicity. However, the cytotoxicity of graphene oxide was greatly mitigated at $10 \%$ FBS, the concentration usually employed in cell medium.

Polyethylene glycol (PEG) conjugation to graphene oxide was examined by several authors. Yang et al. (2011) used nanographene oxide sheets coated with PEG and labeled with radioactive iodine to assess biodistribution and excretion in mice following intravenous injection. These PEG-coated graphene sheets accumulated initially in the liver, and spleen of the mice followed by gradual clearance after 3-5 days. After 3 months, the nanographene sheets were cleared and induced no toxicity at a dose of $20 \mathrm{mg} \mathrm{kg}^{-1}$. PEGcoated nanographene oxide sheets were also prepared by Sun et al. (2008) in order to impart solubility and compatibility of graphene oxide in biological environment. Authors obtained separated PEGylated graphene oxide sheets that selectively recognized and bound to B cell lymphoma cells and were soluble in buffers and serum without agglomeration. 
Moreover, graphene oxide sheets were found to be photoluminescent in the visible and infrared regions. Another study by Liu et al. (2008) showed that graphene is a novel class of material promising for biological applications including future in vivo cancer treatment with various aromatic, low-solubility drugs. Authors functionalized nanographene oxide with branched PEG to obtain a biocompatible graphene oxide-PEG conjugate stable in various biological solutions, and used them for attaching hydrophobic aromatic molecules including a camptothecin analog, SN38, noncovalently via $\pi-\pi$ stacking. The resulting graphene oxide-PEG-SN38 complex exhibited excellent water solubility while maintaining its high in vitro human colon cancer cells killing potency similar to that of the free SN38 molecules in organic solvents. The efficacy of new complex GFM was far higher than that of irinotecan, a FDA-approved water-soluble SN38 prodrug used for the treatment of colon cancer.

The biocompatibility of the functionalized graphene oxide and reduced graphene oxide was analyzed along with the potential biological effects of the used dispersants in L929 mouse fibroblasts by Wojtoniszak et al. (2012). Authors investigated PEG, PEG-polypropylene, glycol-PEG (Pluronic P123), and sodium deoxycholate (DOC) as the dispersants. On the basis on the results of the study, it is possible to conclude that the toxicity depends on the type of dispersant and concentration of the nanomaterials in the suspensions. The best biological properties were observed for graphene oxide functionalized with PEG whereas the other dispersants, i.e., Pluronic 123 and DOC, produced less favorable results. The research indicates that similar to graphene oxide, reduced graphene oxide in PEG is the most biocompatible. The comparison between reduced graphene oxide and graphene oxide shows that the latter has better biocompatibility, especially at higher concentrations such as 50 and $100 \mathrm{~g} \mathrm{ml}^{-1}$. Robinson et al. (2011) tested reduced graphene oxide sheets (with $\sim 20 \mathrm{~nm}$ in average lateral dimension) with high near-infrared (NIR) light absorbance for potential photothermal therapy. The single-layered reduced graphene oxide sheets were functionalized noncovalently by amphiphilic PEGylated polymer chains to render stability in biological solutions. Authors reported that the PEGylated reduced graphene oxide sheets exhibited little toxicity in vitro to human epithelial breast cancer cells at concentrations well above the doses needed for photothermal heating $\left(>80 \mathrm{mg} \mathrm{ml}^{-1}\right)$. Also Gollavelli and Ling (2012) studied in vitro cytotoxicity of multifunction magnetic graphene (coated with PAA and FMA) to human cervical cancer cells (HeLa). Author noted that this form of graphene was non-cytotoxic and did not induce significant amounts of ROS and apoptosis in HeLa cells. In vitro cellular imaging of Multi-function magnetic graphene in HeLa cells revealed sheets localization in the cytoplasmic region of cells without any surface agonist.

Sasidharan et al. (2011) observed the effect of carboxyl functionalization of graphene in pacifying its strong hydrophobic interaction with monkey renal cells and associated toxic effects. Graphene accumulated on the cell membrane causing high oxidative stress leading to apoptosis, whereas carboxyl functionalized hydrophilic graphene was internalized by the cells without causing any toxicity.

Zhang et al. (2011) covalently conjugated graphene oxide with dextran (DEX), a biocompatible polymer widely used for surface coating of biomaterials. Graphene oxide-DEX conjugates demonstrated reduced sheet sizes, increased thickness (TH), and significantly improved stability in physiological solutions. Cellular experiments performed on human cervical cancer HeLa cells showed that DEX coating on graphene oxide remarkably reduced cellular toxicity. Graphene oxide-DEX showed obvious clearance from the mouse body after intravenous injection within a week without causing noticeable short-term toxicity to the treated animals.

\section{Phytotoxicity}

Only one study attempted to address the interactions of graphene or its derivatives with plants. The effects of graphene on root and shoot growth, biomass, shape, cell death, and ROS of cabbage, tomato, red spinach, and lettuce, were analyzed by Begum et al. (2011). The concentrations used in the study ranged from 500 to $2,000 \mathrm{mg} \mathrm{l}^{-1}$. Combined morphological and physiological analyses indicated that after 20 days of exposure under experimental conditions, graphene significantly inhibited plant growth and biomass level. The number and size of leaves of the graphene-treated plants were reduced in a dose-dependent manner. Significant effects were also detected by authors 
showing a concentration-dependent increase in ROS and cell death as well as visible symptoms of necrotic lesions, indicating graphene-induced adverse effects on cabbage, tomato, and red spinach mediated by oxidative stress necrosis. Little or no significant toxic effects were observed with lettuce seedlings under the same conditions. Furthermore, authors also detected the negative effect of graphene on the morphology of roots, finding that the epidermis of the treated tomato and red spinach roots was loosely or completely detached (Begum and Fugetsu 2011).

It should be noted that the plant cell death may occur either by apoptosis or by necrosis which is considered to be the passive mechanism and may be characterized by a progressive loss of membrane integrity resulting in cytoplasmic swelling and release of cellular constituents. The above-mentioned results also indicate that the potential effect of GFM on plants may largely depend on dose, exposure time, and plant species and it deserves further attention.

\section{Anticancer activity}

Relatively few in vitro and in vivo studies concerning GFM anticancer activity have been conducted so far. Feng et al. (2011) reported that at high concentrations (up to $300 \mathrm{~g} \mathrm{ml}^{-1}$ ), polyethyleneimine graphene complexes significantly reduced in vitro toxicity to the treated human epithelial carcinoma (HeLa) cells. Markovic et al. (2011) carried out a comprehensive study on the photothermal anticancer activity of nearinfrared (NIR)-excited graphene. The results suggest that graphene nanoparticles performed significantly better than carbon nanotubes in inducing photothermal death of human glioma (U251) cells in vitro. The mechanisms of graphene-mediated photothermal killing of cancer cells apparently involved oxidative stress and mitochondrial membrane depolarization resulting in mixed apoptotic and necrotic cell death characterized by caspase activation/DNA fragmentation and cell membrane damage, respectively. Similar results were obtained by Zhang et al. (2011). Authors demonstrated that graphene oxide modified with doxorubicin (DOX) and polyethyleneglycol (PEG) during photothermal treatment showed complete in vitro viability reduction in murine mammary tumor (line EMT6) cells as well as in vivo complete destruction of solid tumors (EMT6 tumor-bearing mice were used) without mice weight-loss or recurrence of tumors.

Yang et al. (2010) have also studied the in vivo behaviors of PEGylated nanographene sheets in tumor-bearing mice by in vivo fluorescence imaging. Each mouse was intravenously injected with PEGylated nanographene sheets $\left(200 \mu \mathrm{l}\right.$ of $2 \mathrm{mg} \mathrm{ml}^{-1}$ solution for each mouse at a dose of $20 \mathrm{mg} \mathrm{kg}^{-1}$ ). Authors demonstrated highly efficient tumor passive targeting of graphene sheets in several different tumor models, such as murine breast cancer tumors, human epidermoid carcinoma tumors, and human glioblastoma tumors, without utilizing any targeting ligands, such as antibodies. Thus, PEGylated nanographene sheets appeared to be an excellent in vivo tumor NIR photothermal therapy agent without exhibiting noticeable toxicity to the treated mice.

Zhang et al. (2010) functionalized graphene oxide with sulfonic acid groups, which render it stable in physiological solution, followed by covalent binding of folic acid (FA) molecules to obtain a novel nanocarrier for the loading and targeted delivery of anticancer drugs such as: doxorubicin (DOX) and camptothecin (CPT), onto the FA-conjugated graphene oxide via $p-p$ stacking. Functionalization with folic acid allowed specific targeting of the human breast cancer cells, exhibiting folic acid receptors. Authors demonstrated that FA-graphene oxide loaded with anticancer drugs showed in vitro specific targeting of cancer cells, and remarkably reduced their viability.

\section{Summary}

There have been reported numerous studies focused on GFM biomedical applications. Some of studies regarding the bacterial toxicity of GFM suggest that they may find future application in antimicrobial products. Results suggest that the cell membrane damage of E. coli and $S$. aureus bacteria caused by direct contact of the bacteria with the extremely sharp edges of the nanosheets was the effective mechanism in the bacterial inactivation. However, in contrast to these studies, two studies reported lack of GFM toxicity to E. coli and Shewanella species.

Only a limited number of publications attempted to address the interactions of graphene and its derivatives with living systems. In vitro toxicity investigation 
suggests that GFM exhibit dose-dependent toxicity to mammalian cells (e.g., human lung fibroblasts, epithelial and alveolar epithelial cells, neuronal cells as well as red blood cells), which strongly suggests that their biocompatibility must be considered when GFMs are applied for biomedical engineering. However, only few studies reported biodistribution and toxicity of graphene oxide following intravenous injection in mice. Graphene oxide under low dose did not exhibit obvious toxicity to mice but under high dose exhibited chronic toxicity, causing significant pathological changes, such as granuloma formation, mainly located in the lungs, kidneys, liver, and spleen.

The number of published study results is also greatly limited. The results of studies indicate that GFMs in the free form (highly dispersed and nocoated) exhibit high in vitro cellular toxicity. Nevertheless, GFM in the form of film exhibited good biocompatibility with investigated cells and promoted their growth and proliferation.

Unfortunately, first alarming reports on validity of different toxicity assessment methodologies were found while compiling this review. Liao et al. (2011) discovered that the methylthiazolyldiphenyl-tetrazolium bromide (MTT) assay, a typical nanotoxicity assay failed to predict the toxicity of GFM due to the spontaneous reduction of MTT by GFM, resulting in a false positive signal. Yet, other toxicity assessment, using the watersoluble tetrazolium salt (WST-8) revealed that the investigated GFMs were highly damaging to the investigated cells resulting in acute cytotoxicity. Thus, the usage of MTT assay in predicting the cytotoxicity of GFMs needs to be very careful and other alternate toxicity assays should also be applied according to reliable MTT test results.

Little is also known about toxicity pathways for GFMs. Generation of ROS in target cells is considered to be a potential mechanism for toxicity. The extremely high hydrophobic surface area of graphene may also result in significant biomolecular and cellular interactions with membrane lipids leading to indirect toxicity. GFMs can also produce cytotoxicity in doseand time-dependent means, decreasing cell adhesion, and inducing cell floating and apoptosis. Results indicate that graphene can induce cytotoxicity through the depletion of the mitochondrial membrane potential and the increase of intracellular ROS, and then trigger apoptosis by activation of the mitochondrial pathway. Sadly, the results in the literature are inconsistent, particularly concerning the ability of GFM to enter the cells. While some studies suggest that graphene oxide can enter the cytoplasm and nucleus of human lung epithelial cells and fibroblasts, other studies indicate that graphene oxide cannot enter human alveolar basal epithelial cells. Moreover, cytotoxicity of graphene oxide was found to occur mostly during the initial contact stage of graphene oxide and cells and was independent of exposure duration. Thus, it is possible to draw the conclusion that physical damage of the cell membrane observation excludes the contribution of an oxidative stress mechanism as that is a time-dependent process.

Several studies attempted to address the interactions of graphene or its derivatives with different molecules. There is evidence which suggests that drugs and targeting molecules can be covalently attached to the graphene surface and edge site, or polymers may be adsorbed onto the GFM surface to enhance solubility and biocompatibility. The observations of different molecules related GFM cytotoxicity effects may lead to the creation of an alternative and convenient route to engineer nanomaterials for safe biomedical and environmental applications. The biocompatibility of the functionalized graphene oxide and reduced graphene was analyzed along with the potential biological effects of the used dispersants in cells. The toxicity depended on the type of dispersant and concentration of GFM in the suspensions. Although, different polymeric substrates were used to functionalize GFM for several in vitro and in vivo studies e.g., FBS, PEG derivatives, sodium DOC and DEX, the development of biocompatible surface coating seems to be critical to engineer various functional nanomaterials for biomedical applications. Surface modification of graphene was reported to alter its toxicity, whereas graphene oxide organic conjugates were reported to reduce cellular toxicity to a remarkably higher degree than their native counterparts.

Only one study, so far, attempted to address the interactions of GFMs with plants. The effects of graphene on root and shoot growth, biomass, shape, cell death, and ROS on cabbage, tomato, red spinach, and lettuce were scrutinized. Results suggest that graphene can significantly inhibit plant growth and biomass in a dose- and time-dependent manner. The potential toxic effect of graphene also largely depends on plant species and, thus, should be given much further attention. 


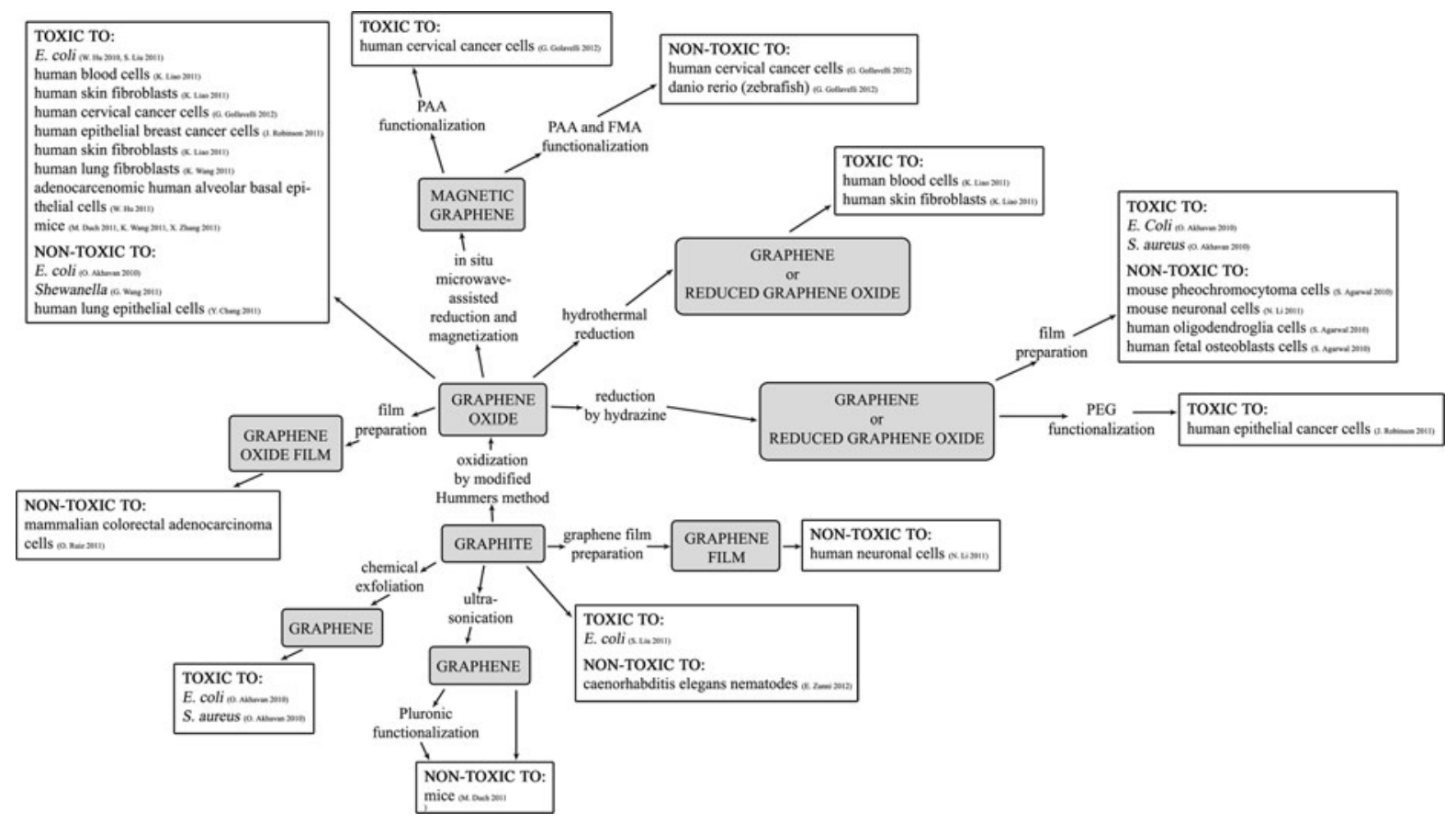

Fig. 2 The schematic summary of the toxicological aspects of graphene family materials in relation to their synthesis techniques

There are relatively few studies concerning GFM anticancer activity. GFMs appeared to be excellent in vitro and in vivo tumor NIR photothermal therapy agents. Significantly reduced viability of in vitro human glioma and human epithelial carcinoma cells was observed without exhibiting noticeable toxicity. Highly efficient tumor passive targeting of GFM has been observed in several different in vivo tumor models without utilizing any targeting ligands, such as antibodies.

What should also be taken into consideration is the fact that GFM synthesis technique determines GFM parameters and their resulting biological activity. In Figs. 2 and 3 the toxicological aspects of GFM in relation to synthesis techniques and resulting properties are presented. It is possible to notice an evident connection between the synthesis technique and bioactivity of GFM (Fig. 2). As has been mentioned before, the discrepancies in toxicological test results may be a result of different toxicity assays used and different sample preparation methods as well as differences in toxicological properties of graphene toward particular investigated cells/organisms. On the other hand, even if the same GFM preparation technique are taken into consideration, it turns out that GFMs are characterized by different properties
(Fig. 3). In this article $\mathrm{TH}$ and lateral dimensions parameters vary the most.

To summarize, the literature on potential health risks of GFM is being published. As for toxicity, a number of studies have been conducted, yet the field still requires further research as it is a newly emerged one and the literature is still greatly limited. The sources are not sufficient to reach conclusions as to potential hazards connected with risk assessment and regulation. The most likely source of the apparent lack of uniformity are different physicochemical properties of GFMs, such as chemical structure, thickness, lateral size, surface charge, surface area, and surface modifications. Undoubtedly, these properties have significant influence on biological/toxicological activity toward investigated cells and animals. However, mentioned GFM parameters are not always wellcontrolled and in some cases even analyzed. Moreover, some of these parameters may also be measured by different techniques, which makes the complied results of studies almost impossible to compare. Consequently, the need for further systematic studies which would address the role of GFM parameters as well as their methods of preparation in determining adverse environmental and health impacts is not emphasized. Furthermore, some guidelines should be drawn by the 
Fig. 3 The schematic summary of the toxicological aspects of graphene oxide in relation to its synthesis techniques and chosen properties such as: thickness (TH) and lateral dimensions (LD)

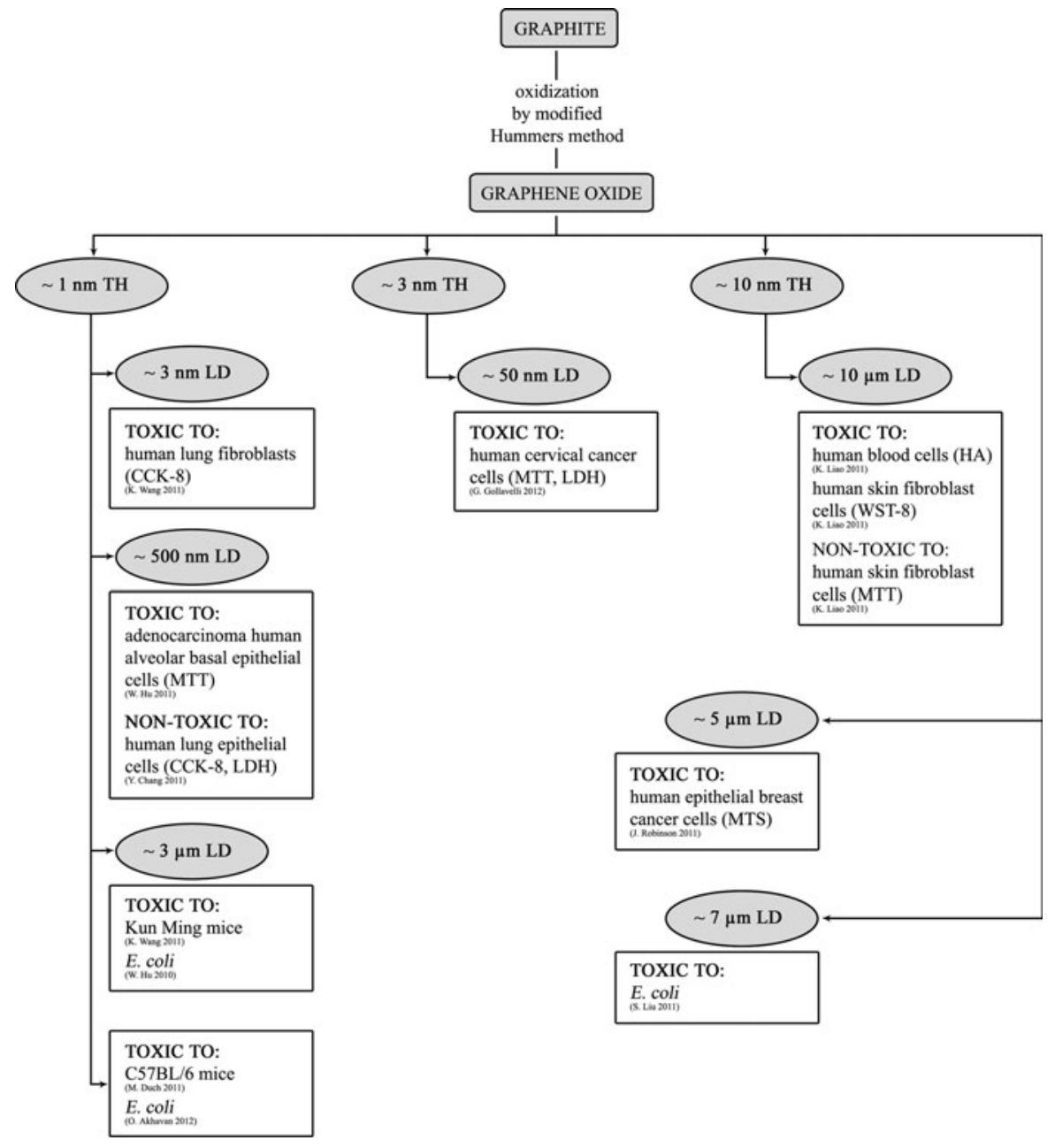

Open Access This article is distributed under the terms of the Creative Commons Attribution License which permits any use, distribution, and reproduction in any medium, provided the original author(s) and the source are credited.

\section{References}

Agarwal S, Zhou X, Ye F, He Q, Chen GC, Soo J, Boey F, Zhang $\mathrm{H}$, Chen $\mathrm{P}$ (2010) Interfacing live cells with nanocarbon substrates. Langmuir 26(4):2244-2247

Akhavan O, Ghaderi E (2010) Toxicity of graphene and graphene oxide nanowalls against bacteria. ACS Nano 10(4):5731-5736

Akhavan O, Ghaderia E (2012) Escherichia coli bacteria reduce graphene oxide to bactericidal graphene in a self-limiting manner. Carbon 50:1853-1860

Begum P, Ikhtiari RI, Fugetsu B (2011) Graphene phytotoxicity in the seedling stage of cabbage, tomato, red spinach, and lettuce. Carbon 49:3907-3919 
Chang Y, Yang ST, Liu JH, Dong E, Wang Y, Cao A, Liu Y, Wang H (2011) In vitro toxicity evaluation of graphene oxide on A549 cells. Toxicol Lett 200:201-210

Duch MC, Budinger GR, Liang YT, Soberanes S, Urich D, Chiarella SE, Campochiaro LA, Gonzalez A, Chandel NS, Hersam MC, Mutlu GM (2011) Minimizing oxidation and stable nanoscale dispersion improves the biocompatibility of graphene in the lung. Nano Lett 11:5201-5207

Feng L, Zhang S, Liu Z (2011) Graphene based gene transfection. Nanoscale 3:1252-1257

Gollavelli G, Ling YC (2012) Multi-functional graphene as an in vitro and in vivo imaging probe. Biomaterials 33: 2532-2545

Hu W, Peng C, Luo W, Lv M, Li X, Li D, Huang Q, Fan C (2010) Graphene-based antibacterial paper. ACS Nano 7(4):4317-4323

Hu W, Peng C, Lv M, Li X, Zhang Y, Chen N, Fan C, Huang Q (2011) Protein corona-mediated mitigation of cytotoxicity of graphene oxide. ACS Nano 5(5):3693-3700

Li N, Zhang X, Song Q, Su R, Qi Z, Kong T, Liu L, Jin G, Tang M, Cheng G (2011a) The promotion of neurite sprouting and outgrowth of mouse hippocampal cells in culture by graphene substrates. Biomaterials 32:9374-9382

Li Y, Zhang P, Du Q, Peng X, Liu T, Wanga Z, Xia Y, Zhang W, Wang K, Zhu H, Wu D (2011b) Adsorption of fluoride from aqueous solution by graphene. J Colloid Interface Sci 363:348-354

Li Y, Liu Y, Fu Y, Wei T, Guyader LL, Gao G, Liu R-S, Chang Y-Z, Chen C (2012) The triggering of apoptosis in macrophages by pristine graphene through the MAPK and TGF-beta signaling pathways. Biomaterials 33(2):402-411

Liao KH, Lin YS, Macosko CW, Haynes CL (2011) Cytotoxicity of graphene oxide and graphene in human erythrocytes and skin fibroblasts. ACS App Mater Interfaces 3: 2607-2615

Liu Z, Robinson JT, Sun XM, Dai HJ (2008) PEGylated nanographene oxide for delivery of water-insoluble cancer drugs. J Am Chem Soc 130(33):10876-10877

Liu S, Zeng TH, Hofmann M, Burcombe E, Wei J, Jiang R, Kong J, Chen Y (2011) Antibacterial activity of graphite, graphite oxide, graphene oxide, and reduced graphene oxide: membrane and oxidative stress. ACS Nano 5(9): 6971-6980

Markovic ZM, Harhaji-Trajkovic LM, Todorovic-Markovic BM, Kepi DP, Arsikin KM, Jovanovi SP, Pantovic AC, Dramicanin MD, Trajkovic VS (2011) In vitro comparison of the photothermal anticancer activity of graphene nanoparticles and carbon nanotubes. Biomaterials 32: $1121-1129$

Neto AC, Peres NMR (2006) Drawing conclusions from graphene. Phys World 19(11):33-37

Neto AC, Guinea F, Peres NM, Novoselov KSS, Geim AK (2009) The electronic properties of graphene. Rev Mod Phys 81:109-162

Novoselov KS, Geim AK, Morozov SV, Jiang D, Zhang Y, Dubonos SV et al (2004) Electric field effect in atomically thin carbon films. Science 306:666-669

Oberdörster G, Oberdörster E, Oberdörster J (2005) Nanotoxicology: an emerging discipline evolving from studies of ultrafine particles. Environmental Health Perspectives 113(7):823-839
Robinson JT, Tabakman SM, Liang Y, Wang H, Casalongue HS, Vinh D, Dai H (2011) Ultrasmall reduced graphene oxide with high near-infrared absorbance for photothermal therapy. J Am Chem Soc 11(133):6825-6831

Ruiz ON, Fernando KA, Wang B, Brown NA, Luo PG, McNamara ND, Vangsness M, Sun YP, Bunker CE (2011) Graphene oxide: a nonspecific enhancer of cellular growth. ACS Nano 5(10):8100-8107

Sanchez VC, Jackhak A, Hurt RH, Kane AB (2011) Biological interactions of graphene-family nanomaterials: an interdisciplinary review. Chem Res Toxicol 25(1):15-34. doi: 10.1021/tx200339h

Sasidharan A, Panchakarla LS, Chandran P, Menon D, Nair S, Rao CNR, Koyakutty M (2011) Differential nano-bio interactions and toxicity effects of pristine versus functionalized graphene. Nanoscale 3(6):2461-2464

Schinwald A, Murphy FA, Jones A, MacNee W, Donaldson K (2012) Graphene-based nanoplatelets: a new risk to the respiratory system as a consequence of their unusual aerodynamic properties. ACS Nano 6(1):736-746

Stone V, Johnston H, Schins RPF (2009) Development of in vitro systems for nanotoxicology: methodological considerations. Crit Rev Toxicol 39:613-626

Sun XM, Liu Z, Welsher K, Robinson JT, Goodwin A, Zaric S (2008) Nano-graphene oxide for cellular imaging and drug delivery. Nano Res 1(3):203-212

Wang G, Qian F, Saltikov CW, Jiao Y, Li Y (2011a) Microbial reduction of graphene oxide by Shewanella. Nano Res 6(4):563-570

Wang K, Ruan J, Song H, Zhang J, Wo Y, Guo S, Cui D (2011b) Biocompatibility of graphene oxide. Nanoscale Res Lett 6:1-8

Wojtoniszak M, Chen X, Kalenczuk RJ, Wajda A, Łapczuk J, Kurzewski M, Drozdzik M, Chu PK, Borowiak-Palen E (2012) Synthesis, dispersion, and cytocompatibility of graphene oxide and reduced graphene oxide. Colloids Surf B 89:79-85

Yan L, Zhao F, Li S, Hu Z, Zhao Y (2011) Low toxic and safe nanomaterials by surface-chemical design, carbon nanotubes, fullerenes, metallofullerenes, and graphenes. Nanoscale 3(2):362-382

Yang K, Zhang S, Zhang G, Sun X, Lee ST, Liu Z (2010) Graphene in mice: ultrahigh in vivo tumor uptake and efficient photothermal therapy. Nano Lett 10:3318-3323

Yang K, Wan J, Zhang S, Zhang Y, Lee ST, Liu Z (2011) In vivo pharmacokinetics, long-term biodistribution, and toxicology of PEGylated graphene in mice. ACS Nano 5:516-522

Yuan J, Gao H, Ching CB (2011) Comparative protein profile of human hepatoma HepG2 cells treated with graphene and single-walled carbon nanotubes: an iTRAQ-coupled 2D LC-MS/MS proteome analysis. Toxicol Lett 207:213-221

Zanni E, De Bellis G, Bracciale MP, Broggi A, Santarelli ML, Sarto MS, Palleschi C, Uccelletti D (2012) Graphite nanoplatelets and Caenorhabditis elegans: insights from an in vivo model. Nano Lett 12:2740-2744

Zhang Y, Mo G, Li X, Zhang W, Zhang J, Ye J, Huang X, Yu C (2011a) A graphene modified anode to improve the performance of microbial fuel cells. J Power Sources 196:5402-5407

Zhang L, Xia J, Zhao Q, Liu L, Zhang Z (2010a) Functional graphene oxide as a nanocarrier for controlled loading and 
targeted delivery of mixed anticancer drugs. Small 6(4): 537-544

Zhang Y, Ali SF, Dervishi E, Xu Y, Li Z, Casciano D, Biris AS (2010b) Cytotoxicity effects of graphene and single-wall carbon nanotubes in neural phaeochromocytoma-derived PC12 cells. ACS Nano 4(6):3181-3186

Zhan X, Yin JL, Peng C, Hu W, Zhu Z, Li W, Fan C, Huang Q (2011a) Distribution and biocompatibility studies of graphene oxide in mice after intravenous administration. Carbon 49:986-995

Zhang S, Yang K, Feng L, Liu Z (2011b) In vitro and in vivo behaviors of dextran functionalized graphene. Carbon 49 : 4040-4049

Zhang W, Guo Z, Huang D, Liu Z, Guo X, Zhong H (2011c) Synergistic effect of chemo-photothermal therapy using PEGylated graphene oxide. Biomaterials 32:8555-8561 